\title{
Initial-stage growth phenomena and distribution of local configurations of the restricted solid-on-solid model
}

\author{
Chih-Chun Chien, ${ }^{1}$ Ning-Ning Pang, ${ }^{1}$ and Wen-Jer Tzeng ${ }^{2, *}$ \\ ${ }^{1}$ Department of Physics, National Taiwan University, Taipei, Taiwan, Republic of China \\ ${ }^{2}$ Department of Physics, Tamkang University, Tamsui, Taipei, Taiwan, Republic of China \\ (Received 20 January 2004; revised manuscript received 22 March 2004; published 11 August 2004)
}

\begin{abstract}
We take a detailed study on the restricted solid-on-solid (RSOS) model with finite nearest-neighbor height difference $S$. We numerically show that, for all finite values of $S$, the system belongs to the random-deposition (RD) class in the early time stage and then crossovers to the Kardar-Parisi-Zhang (KPZ) class. We find that the crossover time scales as $S^{\zeta}$ with the crossover exponent $\zeta=2.06$. Besides, we analytically study the RSOS model by grouping consecutive sites into local configurations to obtain the Markov chain describing the time evolution of the probability distribution of these local configurations. For demonstration, we use the RSOS model with $S=2$ as an explicit example and calculate the correlation functions and even scaling exponents based on the obtained probability distribution of local configurations. The results are very consistent with those obtained from direct simulation of the RSOS model.
\end{abstract}

DOI: 10.1103/PhysRevE.70.021602

PACS number(s): 81.10.Aj, 05.40.-a, 68.55.-a, 02.50.Ey

\section{INTRODUCTION}

Recently, there have been plenty of studies on the phenomenon of kinetic roughening of growing interfaces [1-4]. The comprehension of this phenomenon plays an important role in understanding and controlling a lot of interesting growth processes. Both numerical simulations and real experiments have observed that a large variety of growth processes can be divided into only a few universality classes [1-4]. Each class is characterized by the specific values of the two scaling exponents: the roughness exponent $\alpha$ and the growth exponent $\beta$. Namely, with $h(\mathbf{x}, t)$ denoting the surface height at position $\mathbf{x}$ and time $t$ starting from a flat substrate, the surface width satisfies the dynamic scaling ansatz [5]

$$
w(L, t)=\left\{\overline{\left\langle\left[\overline{(\mathbf{x}, t)-\bar{h}(t)]^{2}}\right\rangle\right.}\right\}^{1 / 2} \sim L^{\alpha} f\left(\frac{t}{L^{z}}\right),
$$

where $L$ is the lateral system size and the scaling function $f(y) \sim y^{\beta}$ for $y \ll 1$ and $f(y) \sim$ constant for $y \gg 1$ with the dynamic exponent $z=\alpha / \beta$. Here and throughout this paper, the overbar denotes spatial average and the angular brackets denote statistical ensemble-average. The correlation function obeys similar dynamic scaling

$$
G(\mathbf{r}, t)=\left\langle\overline{[h(\mathbf{x}+\mathbf{r}, t)-h(\mathbf{x}, t)]^{2}}\right\rangle \sim r^{2 \alpha} g\left(\frac{t}{r^{z}}\right),
$$

where $r=|\mathbf{r}|$ and the scaling function $g(y) \sim y^{2 \beta}$ for $y \ll 1$ and $g(y) \sim$ constant for $y \gg 1$.

Note that the important features of growing surfaces usually can be analyzed and described by some microscopic rules. A number of discrete models for growth phenomena have been proposed and studied successfully by computer simulations. On the other hand, evolution of the growing

*Corresponding author; email address: wjtzeng@mail.tku.edu.tw surface is also, in the coarse-grained sense, described by a continuum equation with additive noise [1-4]. It is generally believed that there is a correspondence between discrete growth models and continuous stochastic Langevin equations. The most common way of establishing the link is to compare the obtained values of the scaling exponents. The other way is to derive the continuum equation from a given discrete model analytically [6,7]. However, the higher order effects are inevitably neglected in these approaches [6,7]. Thus, we expect that the crossover behavior, if any, in the discrete models would be obscure in the corresponding continuum equation obtained from these analytical approaches. Since every approach has its own merit and limitation, any alternative analytical approach to study these growth models will be of particular interest.

Among the various discrete growth models, the restricted solid-on-solid (RSOS) model with finite nearest-neighbor height difference $S$, proposed by Kim and Kosterlitz [8], has drawn much attention for its simplicity and wide applicability, such as the far-from-equilibrium crystal growth at low temperatures [1-4]. For instance, a recent experiment shows that the (001)-surface morphology of GaAs annealed at fixed temperature and pressure can be well explained by the annealed version of the RSOS model by including the nextnearest-neighbor interaction [9]. The study of (001)-oriented GaAs surfaces is technologically important, since it is the starting point for fabricating the majority of high-speed optoelectronic devices and the roughness of surfaces may deteriorate the optical properties of devices. Moreover, even the chemically induced surface roughening occurring through the exothermic release of reaction energy in catalytic reactions on the substrate can also be described in the framework of the RSOS model [10]. In the course of roughening via exothermic catalytic reactions, the reaction energy is transferred to substrate atoms. Thus, it may induce the substrate atoms out of their original sites and consequently create vacancies. Since the roughness of a practical catalyst may effect its global activity, the understanding of such roughening 
phenomena may guide the ways for designing new catalysts and controlling catalytic processes.

It is generally believed that the RSOS model, in the long time and large distance limit, belongs to the universality class of the Kadar-Parisi-Zhang (KPZ) equation [11]. Indeed, the continuum limit of the RSOS model with $S=1$ has been analytically shown $[12,13]$ to belong to the class of the KPZ equation. However, there still lacks extensive studies on the general RSOS model with any possible finite $S$. The singlestep model [14], closely related to the RSOS model, is also believed to belong to the KPZ universality class. Both the single-step model and the $(S=1)$ RSOS model exhibit fast convergence into the KPZ scaling regimes in numerical simulations. Since the height difference between two neighboring sites can only assume two values $(+1$ or -1$)$, the single-step model is mathematically more tractable [15]. In particular, this model can be mapped onto some extensively studied models in equilibrium or nonequilibrium statistical mechanics, such as the kinetic Ising model $[14,16]$, the asymmetric simple exclusion process [17], and the six-vertex model $[14,15]$. Some properties of the single-step model can thus be acquired analytically from the exact results of these well-studied models $[15,16]$. In contrast, less analytical study has been conducted on the RSOS model. In addition, from the experimental point of view, much interest is focused on the very early time aspects of growing interfaces as encountered in actual molecular-beam-epitaxial growth. This motivates us to take an extensive study on the general RSOS model with any possible finite nearest-neighbor height difference $S$.

The outline of this paper is as follows. In Sec. II, the deposition rules of the RSOS model is described and we take an extensive numerical study on the early time behavior of the RSOS model with any finite $S$. In Sec. III, the Markov chain to describe the time evolution of the probability distributions for the local configurations is analytically studied and we use the RSOS model with $S=2$ as an explicit example. Finally, a summary is given in Sec. IV.

\section{INITIAL-STAGE GROWTH PHENOMENA}

We first give the growth rules of the RSOS model as follows:

(1) Choose a site randomly (say, site $x$ ) among all $L$ sites.

(2) If $|[h(x, t)+1]-h(x-1, t)| \leqslant S$ and $\mid[h(x, t]+1)-h(x$ $+1, t \mid \leqslant S$, add $h(x, t)$ by 1 and add $\Delta t(=1 / L)$ to the time.

(3) Otherwise, do nothing.

(4) Repeat the whole process.

Note that, in the original paper of the RSOS model, the authors suggested that the average height of the surface, instead of the number of growth attempts, should be adopted as the simulation time [8].

Since the growth rules of the RSOS model have a parameter $S$, one may wonder how this parameter affects the behavior of the surface of the RSOS model. In the original paper of the RSOS model [8], the authors found that for small $S$, the scaling exponent $\beta$ is unchanged. Thus they claimed that the behavior of the surface of the RSOS model is independent of the parameter $S$. However, if one tries to

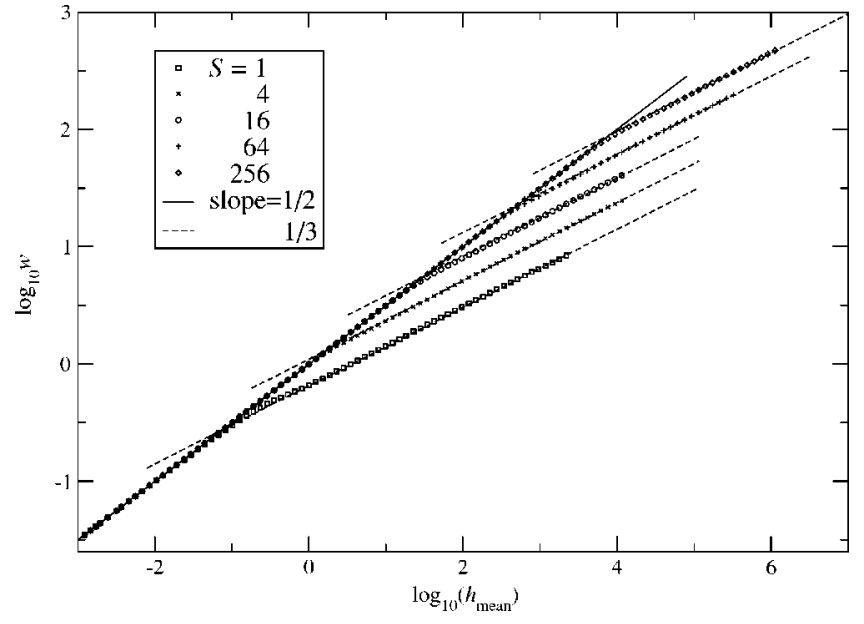

FIG. 1. The log-log plot of the surface width versus the mean surface height in the initial-stage growth process of the general $(1+1)$-dimensional RSOS model with the height difference restriction $S=1,4,16,64,256$, the system size $L=4096$ sites, and averaged over 1000 independent runs.

extend $S$ to infinity, the RSOS model reduces to the random deposition (RD) model. The scaling exponent $\beta$ of the RD model in $(1+1)$-dimensions is $1 / 2$, quite different from that of the $(S=1)$ RSOS model, which is believed to be in the KPZ university class and should have the value $1 / 3$ in (1 +1 )-dimensions. Moreover, we know that the surface width of the $(S=1)$ RSOS model with a substrate of finite size will saturate as the growth time is large enough. This is not the case of the RD model. The surface width of the RD model increases with the growth time $w \sim t^{1 / 2}$ and there is no saturation behavior. In this section, we concentrate on the initialstage growth behavior and look into how the scaling exponent $\beta$ changes its value. In Fig. 1 , we show the results from the computer simulations of the RSOS model in $(1+1)$-dimensions with different height difference restriction $S$ in the early-time stage. We observe that the surface width scales with time (which is the average surface height here) as $w \sim h_{\text {mean }}^{1 / 2}$ at the very early stage and then crossovers to another scaling $w \sim h_{\text {mean }}^{1 / 3}$. For illustration, we draw straight lines with the slope equal to $1 / 2$ and $1 / 3$, respectively, along the data to make the crossover more obvious. The intersection of these straight lines defines the transition point. It indicates that the RSOS model behaves like the RD model in the very early stage of the growth process and, after a duration of time, it "feels" the restrictions on the height differences and turns into the KPZ universality class. We numerically find that the surface width in the initial-stage growth process satisfies the following dynamic scaling ansatz:

$$
w\left(S, h_{\text {mean }}\right)=\tau_{w}(S) f\left(\frac{h_{\text {mean }}}{\tau_{h}(S)}\right)
$$




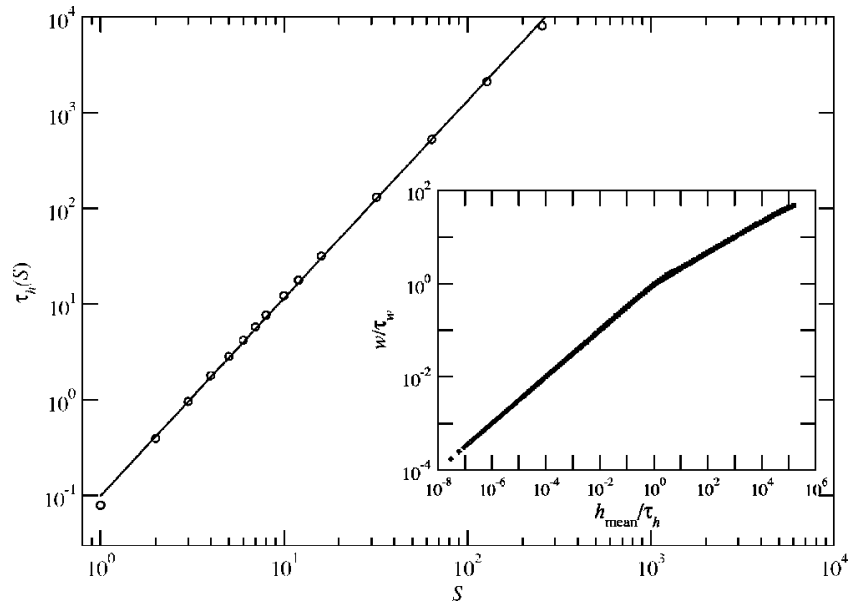

FIG. 2. The log-log plot of the transition mean height, $\tau_{h}(S)$, versus the restriction on the nearest-neighbor height difference $S$ of the general RSOS model in $(1+1)$-dimensions. The straight line is obtained by least squares fit to the data with the crossover exponent equal to 2.06. The inset shows excellent data collapse of the curves from Fig. 1. It confirms that the scaling behavior of the general RSOS model crossovers from the RD regime to the KPZ regime.

$$
f\left(\frac{h_{\text {mean }}}{\tau_{h}(S)}\right) \sim\left\{\begin{array}{l}
{\left[h_{\text {mean }} / \tau_{h}(S)\right]^{1 / 2} \text { for } h_{\text {mean }} \ll \tau_{h}(S),} \\
{\left[h_{\text {mean }} / \tau_{h}(S)\right]^{1 / 3} \text { for } \tau_{h}(S) \ll h_{\text {mean }} \ll L^{z},}
\end{array}\right.
$$

in which $\tau_{h}(S)$ and $\tau_{w}(S)$ denote the transition mean height and the transition surface width, respectively. The inset of Fig. 2 gives the scaling plot, which shows excellent data collapse of the original curves from Fig. 1. In Fig. 2, we also show the scaling relation between the transition mean height $\tau_{h}(S)$ and $S: \tau_{h}(S) \sim S^{\zeta}$ with $\zeta \approx 2.06$. Next, we give a Florytype scaling argument to explain the origin of this scaling behavior. Recall that the RSOS model is just the RD model with restrictions on the height differences. In the beginning of the growth process, there is almost no chance for the neighboring-site height differences larger than $S$ and the behavior of the surface is thus similar to that of the RD model. From the properties of the RD model, we know that the surface width at this stage increases with time (which is the average surface height here) according to $w \sim h_{\text {mean }}^{1 / 2}$. When the surface width is about the value $S$, the surface will "feel" the restrictions and crossover to the KPZ class. So we get a relation between the transition mean height and $S, S$ $\sim \tau_{h}(S)^{1 / 2}$. Thus, the scaling exponent $\zeta=2$. The numerical value of $\zeta$ obtained from the simulation is indeed very close to 2 . In a word, the RSOS model with any finite nearestneighbor height difference $S$ belongs to the KPZ universality class after a long transient time characterized by the scaling exponent $\zeta$ and the RSOS model with $S=\infty$ reduces to the RD model. In comparison, a recent study [18] shows that the RSOS model with any finite hopping distance $l_{0}$ also belongs to the KPZ universality class and the RSOS model with $l_{0}$ $=\infty$ belongs to the universality class of the Villain-Lai-Das Sarma equation [19].

\section{LOCAL CONFIGURATIONS AND PROBABILITY DISTRIBUTIONS}

In the following, the local configurations of the RSOS model and their probability distribution will be investigated in detail. We define the local configurations through the height differences between nearest neighbors, because the absolute height of each site keeps increasing and only the height differences are relevant in determining the success of a growth attempt. By juxtaposing two consecutive height differences, we get a three-site local configuration defined as $K_{x} \equiv\{h(x-1)-h(x), h(x)-h(x+1)\}$ for the one centered at $x$. The three-site local configurations have the property that for each successful growth at site $x$, three and only three local configurations $\left(K_{x-1}, K_{x}\right.$, and $\left.K_{x+1}\right)$ change to new states while all the others remain intact. In this way, the growth problem can be redefined in terms of the finite-state automata [20]. Consideration extended to local configurations with more sites is straightforward.

Next, we will use the $(S=2)$ RSOS model as an explicit example for demonstration. We will analytically obtain the steady-state probability distributions of the local configurations and then calculate the correlation functions and even the scaling exponents based on the obtained probability distributions of local configurations. For the $(S=2)$ RSOS model, the height difference between consecutive sites can assume the values $+2,+1,0,-1$, or -2 and thus there are 25 possible three-site local configurations. The 25 possible local configurations are listed in Table I. $P_{i}(x, t)$ is defined as the probability of the local configuration $K_{x}$ to be of type $i$ at time $t$. Since periodic boundary conditions are implemented, the RSOS model is invariant under spatial translations. Therefore, $P_{i}$ 's are independent of the position and we can drop the argument $x$. Thus, $P_{i}(t)$ may be viewed as the probability of the local configuration, centered at any site, to be

TABLE I. The 25 possible three-site local configurations of the $(S=2)$ RSOS model. $(L, R)$ denotes $[h(x-1)-h(x), h(x)-h(x+1)]$ with the center of the local configuration at site $x$.

\begin{tabular}{lccccccccc}
\hline \hline Type & $(L, R)$ & Type & $(L, R)$ & Type & $(L, R)$ & Type & $(L, R)$ & Type & $(L, R)$ \\
\hline 1 & $(2,2)$ & 2 & $(2,1)$ & 3 & $(2,0)$ & 4 & $(2,-1)$ & 5 & $(2,-2)$ \\
6 & $(1,2)$ & 7 & $(1,1)$ & 8 & $(1,0)$ & 9 & $(1,-1)$ & 10 & $(1,-2)$ \\
11 & $(0,2)$ & 12 & $(0,1)$ & 13 & $(0,0)$ & 14 & $(0,-1)$ & 15 & $(0,-2)$ \\
16 & $(-1,2)$ & 17 & $(-1,1)$ & 18 & $(-1,0)$ & 19 & $(-1,-1)$ & 20 & $(-1,-2)$ \\
21 & $(-2,2)$ & 22 & $(-2,1)$ & 23 & $(-2,0)$ & 24 & $(-2,-1)$ & 25 & $(-2,-2)$ \\
\hline \hline
\end{tabular}


of type $i$ at time $t$. If we know the equations governing the dynamics of all the $P_{i}$ 's, we can study the time evolution and find the asymptotic probability distribution of the local morphology of the system.

Due to the left-right symmetry, we have the following relations among the $P_{i}$ 's:

$$
\begin{gathered}
P_{1}=P_{25}, \quad P_{2}=P_{20}, \quad P_{3}=P_{15}, \quad P_{4}=P_{10}, \quad P_{6}=P_{24}, \\
P_{7}=P_{19}, \quad P_{8}=P_{14}, \quad P_{11}=P_{23}, \quad P_{12}=P_{18}, \quad P_{16}=P_{22} .
\end{gathered}
$$

We may further reduce the number of independent variables by employing the translational symmetry, which gives the following relations:

$$
\begin{aligned}
& P_{2}+P_{3}+P_{4}+P_{5}=P_{6}+P_{11}+P_{16}+P_{21}, \\
& P_{4}+P_{6}+P_{8}+P_{9}=P_{2}+P_{12}+P_{16}+P_{17} .
\end{aligned}
$$

The conservation of probability gives us $\sum_{i} P_{i}=1$, which can be reduced to the following equation with the help of Eq. (5):

$$
\begin{aligned}
& 2\left(P_{1}+P_{2}+P_{3}+P_{4}+P_{6}+P_{7}+P_{8}+P_{11}+P_{12}+P_{16}\right)+P_{5} \\
& \quad+P_{9}+P_{13}+P_{17}+P_{21}=1 .
\end{aligned}
$$

Now we are ready to derive the dynamic equations. The time evolution of the probabilities for local configurations can be described as a Markov process. If we know $\left\{P_{i}(t)\right\}$, the values of the probabilities for local configurations at time $t$, the probabilities after a single growth attempt $\left\{P_{i}(t+\Delta t)\right\}$ can be derived based on the growth rules and the mean-field approximation. Let us begin from the master equation

$$
N_{i}(t+\Delta t)=N_{i}(t)+\sum_{i \neq j}\left[W_{j i}(t)-W_{i j}(t)\right],
$$

where $N_{i}(t)$ denotes the abundance of local configuration of type $i$ at time $t$ with $N_{i}(t)=L P_{i}(t)$ and $W_{i j}(t)$ is the transition probability of the local configuration from type $i$ to type $j$ for a duration $\Delta t=1 / L$ at time $t$. The transition rules are listed in Table II. Since $K_{x-1}$ and $K_{x+1}$ are also affected by a successful growth at site $x$, we need to know the probability distributions for the combinations of $K_{x-1}, K_{x}$, and $K_{x+1}$. Such information should be provided by the probabilities of foursite local configurations. Carrying on such exact analysis, we will face an unlimited input of local configurations of larger sizes and the mathematics will become intractable. Thus, we have to make the mean-field approximation: assuming that the consecutive local configurations are mixed randomly according to their abundance.

This idea is further supported by simulation results in Tables III and IV, as we will explain below. To estimate the value of the roughness exponent $\alpha$, we introduce

$$
\alpha_{r_{1} / r_{2}}=\frac{1}{2} \frac{\ln \left\{G\left(r_{1}\right) / G\left(r_{2}\right)\right\}}{\ln \left(r_{1} / r_{2}\right)}
$$

with $0<r_{1}, r_{2} \ll L$ and $t \rightarrow \infty$. For a random-walk interface with periodic boundary conditions the growth exponent $\alpha$

\begin{tabular}{|c|c|c|c|c|c|}
\hline$K_{x-1}$ & $K_{x}$ & $K_{x+1}$ & $K_{x-1}$ & $K_{x}$ & $K_{x+1}$ \\
\hline $1 \rightarrow 2$ & & $6 \rightarrow 1$ & $1 \rightarrow 2$ & & $11 \rightarrow 6$ \\
\hline $6 \rightarrow 7$ & & $7 \rightarrow 2$ & $6 \rightarrow 7$ & & $12 \rightarrow 7$ \\
\hline $11 \rightarrow 12$ & $2 \rightarrow 6$ & $8 \rightarrow 3$ & $11 \rightarrow 12$ & $3 \rightarrow 7$ & $13 \rightarrow 8$ \\
\hline $16 \rightarrow 17$ & & $9 \rightarrow 4$ & $16 \rightarrow 17$ & & $14 \rightarrow 9$ \\
\hline $21 \longrightarrow 22$ & & $10 \rightarrow 5$ & $21 \rightarrow 22$ & & $15 \rightarrow 10$ \\
\hline $1 \rightarrow 2$ & & $16 \rightarrow 11$ & $1 \rightarrow 2$ & & $21 \rightarrow 16$ \\
\hline $6 \rightarrow 7$ & & $17 \rightarrow 12$ & $6 \rightarrow 7$ & & $22 \rightarrow 17$ \\
\hline $11 \rightarrow 12$ & $4 \rightarrow 8$ & $18 \rightarrow 13$ & $11 \rightarrow 12$ & $5 \rightarrow 9$ & $23 \rightarrow 18$ \\
\hline $16 \rightarrow 17$ & & $19 \rightarrow 14$ & $16 \rightarrow 17$ & & $24 \rightarrow 19$ \\
\hline $21 \rightarrow 22$ & & $20 \rightarrow 15$ & $21 \rightarrow 22$ & & $25 \rightarrow 20$ \\
\hline $2 \rightarrow 3$ & & $6 \rightarrow 1$ & $2 \rightarrow 3$ & & $11 \rightarrow 6$ \\
\hline $7 \rightarrow 8$ & & $7 \rightarrow 2$ & $7 \rightarrow 8$ & & $12 \rightarrow 7$ \\
\hline $12 \rightarrow 13$ & $7 \rightarrow 11$ & $8 \rightarrow 3$ & $12 \rightarrow 13$ & $8 \rightarrow 12$ & $13 \rightarrow 8$ \\
\hline $17 \rightarrow 18$ & & $9 \rightarrow 4$ & $17 \rightarrow 18$ & & $14 \rightarrow 9$ \\
\hline $22 \rightarrow 23$ & & $10 \rightarrow 5$ & $22 \rightarrow 23$ & & $15 \rightarrow 10$ \\
\hline $2 \rightarrow 3$ & & $16 \rightarrow 11$ & $2 \rightarrow 3$ & & $21 \rightarrow 16$ \\
\hline $7 \rightarrow 8$ & & $17 \rightarrow 12$ & $7 \rightarrow 8$ & & $22 \rightarrow 17$ \\
\hline $12 \rightarrow 13$ & $9 \rightarrow 13$ & $18 \rightarrow 13$ & $12 \rightarrow 13$ & $10 \rightarrow 14$ & $23 \rightarrow 18$ \\
\hline $17 \rightarrow 18$ & & $19 \rightarrow 14$ & $17 \rightarrow 18$ & & $24 \rightarrow 19$ \\
\hline $22 \rightarrow 23$ & & $20 \rightarrow 15$ & $22 \rightarrow 23$ & & $25 \rightarrow 20$ \\
\hline $3 \rightarrow 4$ & & $6 \rightarrow 1$ & $3 \rightarrow 4$ & & $11 \rightarrow 6$ \\
\hline $8 \rightarrow 9$ & & $7 \rightarrow 2$ & $8 \rightarrow 9$ & & $12 \rightarrow 7$ \\
\hline $13 \rightarrow 14$ & $12 \rightarrow 16$ & $8 \rightarrow 3$ & $13 \rightarrow 14$ & $13 \rightarrow 17$ & $13 \rightarrow 8$ \\
\hline $18 \rightarrow 19$ & & $9 \rightarrow 4$ & $18 \rightarrow 19$ & & $14 \rightarrow 9$ \\
\hline $23 \rightarrow 24$ & & $10 \rightarrow 5$ & $23 \rightarrow 24$ & & $15 \rightarrow 10$ \\
\hline $3 \rightarrow 4$ & & $16 \rightarrow 11$ & $3 \rightarrow 4$ & & $21 \rightarrow 16$ \\
\hline $8 \rightarrow 9$ & & $17 \rightarrow 12$ & $8 \rightarrow 9$ & & $22 \rightarrow 17$ \\
\hline $13 \rightarrow 14$ & $14 \rightarrow 18$ & $18 \rightarrow 13$ & $13 \rightarrow 14$ & $15 \rightarrow 19$ & $23 \rightarrow 18$ \\
\hline $18 \rightarrow 19$ & & $19 \rightarrow 14$ & $18 \rightarrow 19$ & & $24 \rightarrow 19$ \\
\hline $23 \rightarrow 24$ & & $20 \rightarrow 15$ & $23 \rightarrow 24$ & & $25 \rightarrow 20$ \\
\hline $4 \rightarrow 5$ & & $16 \rightarrow 1$ & $4 \rightarrow 5$ & & $11 \rightarrow 6$ \\
\hline $9 \rightarrow 10$ & & $7 \rightarrow 2$ & $9 \rightarrow 10$ & & $12 \rightarrow 7$ \\
\hline $14 \rightarrow 15$ & $17 \rightarrow 21$ & $8 \rightarrow 3$ & $14 \rightarrow 15$ & $18 \rightarrow 22$ & $13 \rightarrow 8$ \\
\hline $19 \rightarrow 20$ & & $9 \rightarrow 4$ & $19 \rightarrow 20$ & & $14 \rightarrow 9$ \\
\hline $24 \rightarrow 25$ & & $10 \rightarrow 5$ & $24 \rightarrow 25$ & & $15 \rightarrow 10$ \\
\hline $4 \rightarrow 5$ & & $16 \rightarrow 11$ & $4 \rightarrow 5$ & & $21 \rightarrow 16$ \\
\hline $9 \rightarrow 10$ & & $17 \rightarrow 12$ & $9 \rightarrow 10$ & & $22 \rightarrow 17$ \\
\hline $14 \rightarrow 15$ & $19 \rightarrow 23$ & $18 \rightarrow 13$ & $14 \rightarrow 15$ & $20 \rightarrow 24$ & $23 \rightarrow 18$ \\
\hline $19 \rightarrow 20$ & & $19 \rightarrow 14$ & $19 \rightarrow 20$ & & $24 \rightarrow 19$ \\
\hline $24 \rightarrow 25$ & & $20 \rightarrow 15$ & $24 \rightarrow 25$ & & $25 \rightarrow 20$ \\
\hline
\end{tabular}
has been shown to be 1/2 [21]. Except for the periodic boundary conditions, the height differences at difference
TABLE II. Rules of changes of local configurations of the $(S=2)$ RSOS model when an attempt of growth on site $x$ is made. $K_{x-1}, K_{x+1}$, and $K_{x}$ will change to new states simultaneously according to the given rules. Note that for each given $K_{x}$, there are five possibilities for $K_{x-1}$ and $K_{x+1}$, respectively.

places in a random-walk interface are statistically independent and we expect $\alpha_{r_{1} / r_{2}}$ to be very close to $1 / 2$ for any $r_{1}, r_{2} \ll L$. From the growth rules of the RSOS model, we also expect that the correlations of the height differences are mainly of short ranges and therefore the values of $\alpha_{r_{1} / r_{2}}$ 
TABLE III. Simulation results of the exponent $\alpha_{r_{1} / r_{2}}$ defined in Eq. (9) for the $(S=2)$ RSOS model with $L=4000$ sites, $t=5 \times 10^{5}$ monolayers, and averaged over 600 independent runs.

\begin{tabular}{lcccc}
\hline \hline$\alpha_{2 / 1}$ & $\alpha_{4 / 2}$ & $\alpha_{8 / 4}$ & $\alpha_{16 / 8}$ & $\alpha_{32 / 16}$ \\
\hline 0.572 & 0.5572 & 0.539 & 0.522 & 0.509 \\
\hline \hline
\end{tabular}

should converge to $1 / 2$ for increasing $r_{1}$ and $r_{2}$ under the condition $r_{1}, r_{2} \ll L$. As illustrated in Table III, the convergence of $\alpha$ is indeed very fast and the deviation is within $15 \%$ even for the lowest possible values of $r_{1}$ and $r_{2}$. To justify our conjecture for the range of the correlations, we also measure the height-difference correlations, defined as

$$
C_{\Delta h}(r)=\langle\overline{[h(x+r)-h(x+r-1)][h(x)-h(x-1)]}\rangle .
$$

The correlation of the height differences is indeed mainly from the interactions of the nearest neighbors, as illustrated in Table IV.

The flow equations governing the evolutions of the $P_{i}$ 's are derived from the general formula Eq. (8). After we write down the form of each $W_{i j}$ with the help of Table II and collect the nonvanishing terms, we obtain the whole set of flow equations for $\left\{P_{i}(t), \forall i\right\}$. The explicit expressions of these flow equations are given in the Appendix. We may study the stationary behavior of these equations by setting $\Delta P_{i}(t) / \Delta t=0, \forall i$. The asymptotic solution obtained by solving the set of the stationary equations with the help of Eqs. (6) and (7) are listed in Table V. In that table, we also list the numerical results obtained from computer simulation of the $(S=2)$ RSOS model. Comparing these two results, we see that our method gives quite accurate predictions of the probability distribution for the local configurations of the
TABLE IV. Simulation results of the height-difference correlations $C_{\Delta h}(r)$ defined in Eq. (10) for the $(S=2)$ RSOS model with $L=4000$ sites, $t=5 \times 10^{5}$ monolayers, and averaged over 600 independent runs.

\begin{tabular}{lcccc}
\hline \hline$r$ & 1 & 2 & 3 & 4 \\
\hline$C_{\Delta h}(r)$ & 0.220 & 0.0654 & 0.0293 & 0.0165 \\
\hline \hline
\end{tabular}

$(S=2)$ RSOS model and the relative deviation is within $6 \%$.

From the obtained results, we are able to calculate the correlation function. For example,

$$
G(r=1, t \rightarrow \infty)=\sum_{i} P_{i}(t \rightarrow \infty)\left\{[h(x+1)-h(x)]_{i}\right\}^{2},
$$

where $[h(x+1)-h(x)]_{i}$ denotes the quantity for the local configuration of type $i$. For the value of $G(r \geqslant 3, t \rightarrow \infty)$, one may "augment" each type of local configuration with their possible neighbors according to relative probabilities in the spirit of the mean-field approximation. Then, we can build a new set of local configurations with larger sizes and obtain the values of $G(r \geqslant 3, t \rightarrow \infty)$. We may proceed to obtain the growth exponent $\alpha_{r_{1}} / r_{2}$, defined in Eq. (9). The values of $\alpha_{r_{1} / r_{2}}$ from the analytical calculation and from the direct simulation of the $(S=2)$ RSOS model are both listed in Table VI for comparison. Note that, even with the device of "augmentation" used in analytical calculations of $\alpha_{3 / 2}$ and $\alpha_{4 / 3}$, the results are still quite close to those from direct simulation of the $(S=2)$ RSOS model. The results from analytical calculations and those from computer simulations both show the tendency that $\alpha_{r_{1} / r_{2}}$ converges toward $1 / 2$. Subsequently, by iteration of Eq. (8) from $t=0$, we can obtain the time

TABLE V. The steady state probability distribution for the local configurations of the $(S=2)$ RSOS model. The data are obtained from solving the steady state solution of Eqs. (12) to (26) and from direct computer simulations of the $(S=2)$ RSOS model with $L=8000$ sites, $t=10^{5}$ monolayers, and averaged over 500 independent runs.

\begin{tabular}{lccccc}
\hline \hline & $P_{1}$ & $P_{2}$ & $P_{3}$ & $P_{4}$ & $P_{5}$ \\
\hline Steady state solution & 0.0510 & 0.0401 & 0.0339 & 0.0320 & 0.0513 \\
Simulation & 0.0542 & 0.0412 & 0.0338 & 0.0321 & 0.0493 \\
\cline { 2 - 5 } & $P_{6}$ & $P_{7}$ & $P_{8}$ & $P_{9}$ & $P_{10}$ \\
\cline { 2 - 5 } Steady state solution & 0.0479 & 0.0397 & 0.0357 & 0.0406 & 0.0320 \\
Simulation & 0.0489 & 0.0391 & 0.0353 & 0.0390 & 0.0321 \\
\cline { 2 - 5 } & $P_{11}$ & $P_{12}$ & $P_{13}$ & $P_{14}$ & $P_{15}$ \\
Steady state solution & 0.0453 & 0.0392 & 0.0377 & 0.0357 & 0.0339 \\
Simulation & 0.0447 & 0.0385 & 0.0379 & 0.0353 & 0.0338 \\
\cline { 2 - 5 } & $P_{16}$ & $P_{17}$ & $P_{18}$ & $P_{19}$ & $P_{20}$ \\
Steady state solution & 0.0386 & 0.0383 & 0.0392 & 0.0397 & 0.0401 \\
Simulation & 0.0379 & 0.0376 & 0.0385 & 0.0391 & 0.0412 \\
\cline { 2 - 5 } & $P_{21}$ & $P_{22}$ & $P_{23}$ & $P_{24}$ & $P_{25}$ \\
Steady state solution & 0.0253 & 0.0386 & 0.0453 & 0.0479 & 0.0510 \\
Simulation & 0.0249 & 0.0379 & 0.0447 & 0.0489 & 0.0542 \\
\hline \hline
\end{tabular}


TABLE VI. The growth exponent $\alpha_{r_{1} / r_{2}}$ defined in Eq. (9) for the $(S=2)$ RSOS model. Data are obtained from two different methods: analytical calculations and computer simulations with $L$ $=4000$ sites, $t=10^{5}$ monolayers, and averaged over $10^{3}$ independent runs.

\begin{tabular}{lccc}
\hline \hline & $\alpha_{2 / 1}$ & $\alpha_{3 / 2}$ & $\alpha_{4 / 3}$ \\
\hline Analytical calculation & 0.558 & 0.532 & 0.522 \\
Computer simulation & 0.572 & 0.561 & 0.551 \\
\hline \hline
\end{tabular}

evolution of $P_{i}$ 's (the probability distributions for local configurations) and then perform the scaling plot of $G(r, t) / r^{2 \alpha}$ versus $t / r^{z}$ to estimate the dynamical exponent $z$. Figure 3 shows the excellent data collapse of $G(r=1, t)$ and $G(r$ $=2, t)$ for $\alpha_{2 / 1}=0.558$ and $z=2-\alpha_{2 / 1}=1.442$. We do not include $G(r, t)$ with $r>2$ in the scaling plot of Fig. 3, since the values of $G(r, t)$ with $r>2$ are obtained through the operation of "augmentation" in our mean-field approximation and thus assume their saturation values as soon as all the $P_{i}(t)$ 's reach saturations. Solely from the local configurations of the RSOS model, we have successfully obtained the growth exponents $\alpha$ and $z$. In this way, we demonstrate the effectiveness of this approach for studying interfacial roughening phenomena.

\section{CONCLUSION}

Recently, there have been plenty of studies on the phenomenon of kinetic roughening of growing interfaces, such as advances of bacterial colonies, electrochemical deposition, flameless fire fronts, and molecular-beam-epitaxial growth [1-4]. The comprehension of this phenomenon plays an important role in understanding and controlling a lot of interesting growth processes and thus is technologically important. For example, the development of thin film roughness of the optoelectronic devices has great influence on their op-

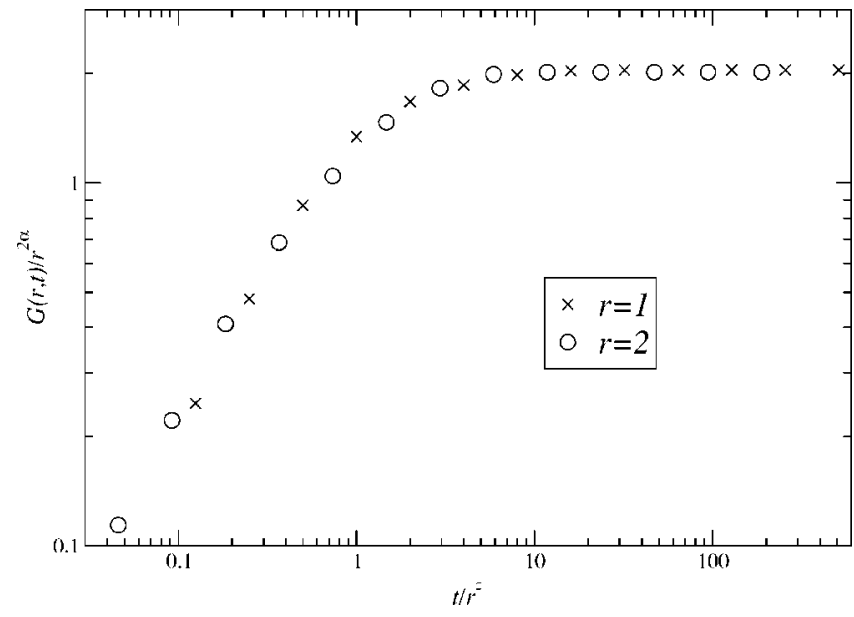

FIG. 3. The scaling plot of $G(r, t)$ obtained from our local configuration approach with $r=1$ and 2 for the $(S=2)$ RSOS model. It shows excellent data collapse with $\alpha_{2 / 1}=0.558$ and $z=2-\alpha_{2 / 1}$ $=1.442$. tical properties due to the increasing number of the scattering centers. Among the various discrete growth models, the RSOS model with finite nearest-neighbor height difference $S$, proposed by Kim and Kosterlitz, has drawn much attention for its simplicity and wide applicability. It is believed that this model belongs to the KPZ universality class in the continuum limit. Due to the simplicity of its growth algorithm, it has been used as a template for modeling many physical and chemical processes, e.g., the surface roughening via exothermic catalytic reactions on the substrate.

However, the past studies in the literature all focus on the RSOS model with $S=1$. Thus, we are motivated to take a detailed numerical study on the RSOS model with arbitraty nearest-neighbor height difference $S$. We numerically show that, for all finite values of $S$, the system belongs to the RD class in the early time stage and then crossovers to the KPZ class. We find that the crossover time scales as $S^{\zeta}$ with the numerically measured crossover exponent $\zeta=2.06$. Then, a solid physical argument is given to explain the crossover phenomenon and the value of the crossover exponent. This is exactly the origin of the so-called "intrinsic width" in the very early time stage of the growth. Our argument can be extended to explain the origin of the intrinsic width in other stochastic growth models such as the Family model [22], the Wolf-Villain model [23], and the Das Sarma-Tamborenea model [24], etc. The generalization to higher dimensions is straightforward and we believe that the crossover exponent $\zeta=2$ is independent of dimensionality.

Besides, we propose an alternative analytical approach to study the local stochastic growth models, by grouping consecutive sites into local configurations and then deriving the time evolution of the probability distribution of these local configurations. For demonstration, we use the RSOS model with $S=2$ as an example and explicitly obtain the probability distribution of local configurations. Based on the obtained results, we then derive the correlation functions and even the scaling exponents $\alpha$ and $z$, which are consistent very well with those obtained from direct simulation of the RSOS model or the KPZ equation. Note that the values of $G(r>2, t)$ are obtained through the operation of "augmentation" in our mean-field approach and thus should not be used to obtain the dynamics of the system. Only with the information of $G(r=1, t)$ and $G(r=2, t)$, we are able to determine the values of the scaling exponents, which fully confirm the scaling relation $\alpha+z=2$. In this way, we demonstrate the feasibility and the effectiveness of this alternative approach for studying the interfacial roughening phenomena governed by local growth rules.

\section{ACKNOWLEDGMENTS}

The work of N.-N. P. is supported in part by the National Science Council of the Republic of China under Grant No. NSC-92-2112-M002-017. The work of W.-J. T. is supported in part by the National Science Council of the Republic of China under Grant No. NSC-92-2112-M032-011.

\section{APPENDIX}

Here, we will give the explicit expressions of the flow equations for $\left\{P_{i}(t), \forall i\right\}$. With the help of Eq. (5), we only 
need to list out the flow equations for $\left\{P_{i}(t), i\right.$ $=1, \ldots, 9,11,12,13,16,17,21\}$.

$$
\begin{aligned}
& \frac{\Delta P_{1}}{\Delta t}=\frac{P_{6}\left(P_{2}+P_{7}+P_{12}+P_{17}\right)}{P_{4}+P_{6}+P_{7}+P_{8}+P_{9}} \\
& -\frac{P_{1}\left(P_{2}+P_{3}+P_{4}+P_{5}\right)}{P_{1}+P_{6}+P_{11}+P_{16}+P_{21}}, \\
& \frac{\Delta P_{2}}{\Delta t}=-P_{2}+\frac{P_{1}\left(P_{2}+P_{3}+P_{4}+P_{5}\right)}{P_{1}+P_{6}+P_{11}+P_{16}+P_{21}} \\
& +\frac{P_{7}\left(P_{2}+P_{7}+P_{12}+P_{17}\right)}{P_{4}+P_{6}+P_{7}+P_{8}+P_{9}} \\
& -\frac{P_{2}\left(P_{4}+P_{7}+P_{8}+P_{9}\right)}{P_{2}+P_{7}+P_{12}+P_{16}+P_{17}}, \\
& \frac{\Delta P_{3}}{\Delta t}=-2 P_{3}+\frac{P_{3} P_{11}}{P_{3}+P_{8}+P_{11}+P_{12}+P_{13}} \\
& +\frac{P_{8}\left(P_{2}+P_{7}+P_{12}+P_{17}\right)}{P_{4}+P_{6}+P_{7}+P_{8}+P_{9}} \\
& +\frac{P_{2}\left(P_{4}+P_{7}+P_{8}+P_{9}\right)}{P_{2}+P_{7}+P_{12}+P_{16}+P_{17}}, \\
& \frac{\Delta P_{4}}{\Delta t}=P_{3}-P_{4}+\frac{\left(P_{9}-P_{4}\right)\left(P_{2}+P_{7}+P_{12}+P_{17}\right)}{P_{4}+P_{6}+P_{7}+P_{8}+P_{9}} \\
& -\frac{P_{11} P_{3}}{P_{3}+P_{8}+P_{11}+P_{12}+P_{13}} \text {, } \\
& \frac{\Delta P_{5}}{\Delta t}=-P_{5}+\frac{2 P_{4}\left(P_{2}+P_{7}+P_{12}+P_{17}\right)}{P_{4}+P_{6}+P_{7}+P_{8}+P_{9}}, \\
& \frac{\Delta P_{6}}{\Delta t}=P_{2}+P_{11}-\frac{P_{6}\left(P_{2}+P_{3}+P_{4}+P_{5}\right)}{P_{1}+P_{6}+P_{11}+P_{16}+P_{21}} \\
& -\frac{P_{6}\left(P_{2}+P_{7}+P_{12}+P_{17}\right)}{P_{4}+P_{6}+P_{7}+P_{8}+P_{9}} \\
& -\frac{P_{11} P_{11}}{P_{3}+P_{8}+P_{11}+P_{12}+P_{13}} \\
& \frac{\Delta P_{7}}{\Delta t}=P_{3}-P_{7}+P_{12}-\frac{P_{11} P_{12}}{P_{3}+P_{8}+P_{11}+P_{12}+P_{13}} \\
& -\frac{P_{6}\left(P_{2}+P_{3}+P_{4}+P_{5}\right)}{P_{1}+P_{6}+P_{11}+P_{16}+P_{21}} \\
& -\frac{P_{7}\left(P_{2}+P_{7}+P_{12}+P_{17}\right)}{P_{4}+P_{6}+P_{7}+P_{8}+P_{9}} \\
& -\frac{P_{7}\left(P_{4}+P_{7}+P_{8}+P_{9}\right)}{P_{2}+P_{7}+P_{12}+P_{16}+P_{17}},
\end{aligned}
$$

$$
\begin{aligned}
\frac{\Delta P_{8}}{\Delta t}= & P_{4}-2 P_{8}+P_{13}-\frac{P_{11}\left(P_{13}-P_{8}\right)}{P_{3}+P_{8}+P_{11}+P_{12}+P_{13}} \\
& -\frac{P_{8}\left(P_{2}+P_{7}+P_{12}+P_{17}\right)}{P_{4}+P_{6}+P_{7}+P_{8}+P_{9}} \\
& -\frac{P_{7}\left(P_{4}+P_{7}+P_{8}+P_{9}\right)}{P_{2}+P_{7}+P_{12}+P_{16}+P_{17}},
\end{aligned}
$$

$$
\begin{aligned}
\frac{\Delta P_{9}}{\Delta t}= & P_{5}-P_{9}+2 P_{8}-\frac{2 P_{11} P_{8}}{P_{3}+P_{8}+P_{11}+P_{12}+P_{13}} \\
& -\frac{2 P_{9}\left(P_{2}+P_{7}+P_{12}+P_{17}\right)}{P_{4}+P_{6}+P_{7}+P_{8}+P_{9}},
\end{aligned}
$$

$$
\begin{aligned}
\frac{\Delta P_{11}}{\Delta t}= & P_{7}-P_{11}+\frac{P_{11} P_{11}}{P_{3}+P_{8}+P_{11}+P_{12}+P_{13}} \\
& -\frac{P_{11}\left(P_{2}+P_{3}+P_{4}+P_{5}\right)}{P_{1}+P_{6}+P_{11}+P_{16}+P_{21}} \\
& +\frac{P_{16}\left(P_{4}+P_{7}+P_{8}+P_{9}\right)}{P_{2}+P_{7}+P_{12}+P_{16}+P_{17}},
\end{aligned}
$$

$$
\begin{aligned}
\frac{\Delta P_{12}}{\Delta t}= & P_{8}-2 P_{12}+\frac{P_{11} P_{12}}{P_{3}+P_{8}+P_{11}+P_{12}+P_{13}} \\
& +\frac{P_{11}\left(P_{2}+P_{3}+P_{4}+P_{5}\right)}{P_{1}+P_{6}+P_{11}+P_{16}+P_{21}} \\
& +\frac{\left(P_{17}-P_{12}\right)\left(P_{4}+P_{7}+P_{8}+P_{9}\right)}{P_{2}+P_{7}+P_{12}+P_{16}+P_{17}},
\end{aligned}
$$

$$
\begin{aligned}
\frac{\Delta P_{13}}{\Delta t}= & P_{6}-3 P_{13}+\frac{2 P_{11} P_{13}}{P_{3}+P_{8}+P_{11}+P_{12}+P_{13}} \\
& +\frac{2 P_{12}\left(P_{4}+P_{7}+P_{8}+P_{9}\right)}{P_{2}+P_{7}+P_{12}+P_{16}+P_{17}},
\end{aligned}
$$$$
\frac{\Delta P_{16}}{\Delta t}=P_{12}+\frac{\left(P_{21}-P_{16}\right)\left(P_{2}+P_{3}+P_{4}+P_{5}\right)}{P_{1}+P_{6}+P_{11}+P_{16}+P_{21}}
$$$$
-\frac{P_{16}\left(P_{4}+P_{7}+P_{8}+P_{9}\right)}{P_{2}+P_{7}+P_{12}+P_{16}+P_{17}},
$$

$$
\begin{aligned}
\frac{\Delta P_{17}}{\Delta t}= & P_{13}-P_{17}+\frac{2 P_{16}\left(P_{2}+P_{3}+P_{4}+P_{5}\right)}{P_{1}+P_{6}+P_{11}+P_{16}+P_{21}} \\
& -\frac{2 P_{17}\left(P_{4}+P_{7}+P_{8}+P_{9}\right)}{P_{2}+P_{7}+P_{12}+P_{16}+P_{17}}
\end{aligned}
$$$$
\frac{\Delta P_{21}}{\Delta t}=P_{17}-\frac{2 P_{21}\left(P_{2}+P_{3}+P_{4}+P_{5}\right)}{P_{1}+P_{6}+P_{11}+P_{16}+P_{21}} .
$$ 
[1] A.-L. Barabási and H. E. Stanley, Fractal Concepts in Surface Growth (Cambridge University Press, Cambridge, 1995).

[2] Surface Disordering: Growth, Roughening, and Phase Transitions, edited by R. Jullien, J. Kortesz, P. Meakin, and D. E. Wolf (Nova Science, New York, 1992)

[3] T. Halpin-Healy and Y.-C. Zhang, Phys. Rep. 254, 215 (1995).

[4] A. Pimpinelli and J. Villain, Physics of Crystal Growth (Cambridge University Press, Cambridge, 1998).

[5] F. Family and T. Vicsek, J. Phys. A 18, L75 (1985).

[6] D. D. Vvedensky, A. Zangwill, C. N. Luse, and M. R. Wilby, Phys. Rev. E 48, 852 (1993).

[7] G. Costanza, Phys. Rev. E 55, 6501 (1997).

[8] J. M. Kim and J. M. Kosterlitz, Phys. Rev. Lett. 62, 2289 (1989).

[9] Z. Ding, D. W. Bullock, P. M. Thibado, V. P. LaBella, and K. Mullen, Phys. Rev. Lett. 90, 216109 (2003).

[10] V. P. Zhdanov and B. Kasemo, Surf. Sci. 418, 84 (1998).

[11] M. Kardar, G. Parisi, and Y. C. Zhang, Phys. Rev. Lett. 56, 889 (1986).

[12] K. Park and B. N. Kahng, Phys. Rev. E 51, 796 (1995).
[13] Z. F. Huang and B. L. Gu, Phys. Rev. E 57, 4480 (1998).

[14] P. Meakin, P. Ramanlal, L. Sander, and R. C. Ball, Phys. Rev. A 34, 5091 (1986).

[15] J. Neergaard and M. den Nijs, J. Phys. A 30, 1935 (1997).

[16] M. Plischke, Z. Rácz, and D. Liu, Phys. Rev. B 35, 3485 (1987)

[17] B. Derrida, Phys. Rep. 301, 65 (1998).

[18] S.-C. Park, J.-M. Park, and D. Kim, Phys. Rev. E 65, 036108 (2002).

[19] J. Villain, J. Phys. I 1, 19 (1991);Z.-W. Lai and S. Das Sarma, Phys. Rev. Lett. 66, 2348 (1991).

[20] M. Sipser, Introduction to the Theory of Computation (PWS Publishing Company, Boston, 1997).

[21] G. Foltin, K. Oerding, Z. Rácz, R. L. Workman, and R. K. P. Zia, Phys. Rev. E 50, 639 (1994).

[22] F. Family, J. Phys. A 19, L441 (1986).

[23] D. E. Wolf and J. Villain, Europhys. Lett. 13, 389 (1990).

[24] S. Das Sarma and P. Tamborenea, Phys. Rev. Lett. 66, 325 (1991). 